Ophthalmologica 1949;118:212-214

\title{
Contents, Vol. 118, No. 4-5, 1949
}

\section{INDEX}

Dioptrik un $<1$ I'hj siologische Optik

Die oplische Wirkungsweise dcs Haftglases und ihre Bedeutung für die Ophthalmologie. Von E. Bürki - Basel 215

* $\quad$ Prin/.ip und Phänomene der Schattenprobe. Von O. Rappaport - Batavia

234Riologise.hes zum Horopterproblem. Von W.P.C.Zeeman - Amsterdam

254Líeber < las entoptisehe Phänomen der «blauen Bögen». Von G. Böhm - Basel

276Sur queSques phénomènes entoptiques. Par A. Truntus - Athènes . . . 305' ' Eeber die Purkinje'schen Aderfiguren und die Doppelbeleuclitung in

O'phthalmoskopie u. Spaitlampenuntersucbung. Von E. 1·L·ruupu - Liberee 318

L·eber die Wirkung einiger Pharmaka auf die Nachbilddauer. Von J. Ar-

benz - Basel 321

Iliophysik und Biochemie

Filme und Phasengrenzen im Auge. Von F. P. Fischer $\uparrow-$ Utrecht . .

Untersuchungen über «alkalische» Phosphatase in der

Cornea. Von H. Süllmunn und P. Payot - Basel

335Histocheinische

' $\square$ ' Mici-odosagc de $\Gamma$ Azote Proiéinique dans Thumeur aqueuse. Par C. Bula-

voine el N. Vuataz - (ienève 356

Le durcissement c! u cristallin. Par ]. Nordmunn — Strasbourg ....

Ophthalmo-Neurologie; Angenbewegungen

Komplette Blieklähmung in einem Falle von Heine-Medinscher Polio-Encephalitis acuta. Von M. Pfluglelder und F. Hawser Basel . . 378 Hémianopsie et nystagmus optocinétique. Par W.Stadlin - Geneve . . 383 i unktion und Funktionsstörung des M. Obliquus superior. Von C.O.Roe-

lofs - Amsterdam $\quad, \quad 389$

Cornea

* Experimentelle Studien /, ur Patho-physiologie des Hornhautepithels: Zell-

Bewegungen bei der Wundheilung, Zell-Teilung, Mitoseîiemniung undandere Kernphänomene. Von W.Buschke - New York 407

A New Method for Studying Endothelial Regeneration. By D. G. Cogan Boston 440

Aids to Cornea] Staining. By J.R.Anderson a Melbourne 444

llvea

Dunkelfcld- und ultraniikroskopische Aspekte des frischen Kammerwassers. Von M. Amsler und A. Huber $\square$ Zurich 450

Uvéite antérieure aiguë à staphylocoques dorés. Par F.Verrey - Zurich 461

The Part Played by Roentgen Rays in the Treatment of Uveitis. By $\Lambda$ í. E. Alvaro - Sao Paulo 469

Considerations anciennes et nouvelles sur la pathologie et la pathogénie 
de Гophtalmie sympathique. Par E. Redslob - Strasbourg .... 483

AugendVuek und Glaukom

Die Kamn.erwasservenen und das Poiseuille'sche (iesetz. Von H. Gold-

mann-Bern 496

Index

213

Beitrag zur physikalischen und pharmakologisohen Beeinflussung des

Augendruckes. Von R. Bruckner, C. Hermann und S. Jent-Peyer - Basel 520

Contribution a la neuroclinique du glaucome. Par J. Sedan et S. Sedan-

Bauby - Marseille 534

L'hypopituitai isme dans cinq eas de glaucome juvenile primitif. Par

P. Jeandelize - Nancy 548

Les modifications de la frequence critique de fusion dans le glaucome.

Par R. Weekers, F. Roussel et A. Heíntz - Liége ...... 555

Mode d'action des operations antiglaucomateuses. Par L. Weekers - Liége 564

Retina

La Rubeosis iridis. Par P. Bonnet - Lyon 57.:

Le tonus des artères rétmiennes. Par P. Baílliart - Paris 589

Les progrès realises dans $\Gamma$ étude de la circulation sanguine rétinienne au cours des dernières années. Par A. Fritz - Bruxelles 597

$3 / 8$ Zur Genese der Retina- und Opticus-Scheidenblu \mgen bei akuter intra-kranieller

Drucksteigerung. Von R. Bruckner, W. Bloch und L. Wolff-W' iesinger - Basel 607

* New-formed Vessels in the Fundus Oculi. By A. J. Ballantyne - Glasgow . 611Xanthosis

(vel discoloratio flava) Fundi Diabetica and the Appearance of

«Sharply Outlined* Retinal Vessels - Hitherto Unnoticed Characteristic Features in Diabetic

Retinopathy. By A. Feigenbaum - Jerusalem 632 Ueber Retinitis pigmentosa und innere

Sekretion. Von H. Hegner - Basel 637

* $\quad$ Ein Fall von doppelseitiger Ablatio retinae bei nephrogener Neuroretinitis

angiospastica. Von H. Gerber - Wien

645

Quelques considerations sur le décollement de la réti $\pi$ e. Par G. F. Cosmet-

tatos - Athènes

651

Quelques deductions à propos de deux mille eas de décollement de rétine

opérés. Par îi. Amiga - Barcelone 655

* Bulbusverkürzung durch Reffung der Sclera. Von H. Weve - Utrecht . 660

Infektionsk rankheiten und Intoxikationen

* Conjunctivitis pseudomembranacea chronica granulomatosa lignosa. Von

G.Suubermann - Basel 666

Sur Гétiologie du trachome. Par R. Nutaf - Tunis 687

La chorio-rétinite en «taches de bougie», manifestation de la maladie de

Besnier-Boeck. Par A. Franceschettí et /. Babel @ Geneve .... 701

* Das Vorkommen von Uveitis bei congenitaler bzw. frühinfantiler Me-

ningo-Encephalo-Myelitís. Von C. D. Binkhorst - Terneuzen, L. M. C.

Bedaux $\uparrow-$ Bergen op Zoom, W. G. Mt van der Valk — Bergen op

Zoom, P.K. de Haas - Goes und R. Bruckner - Basel 711

Les troubles oculaires au cours de revolution de la trypanosomiase hu-

maine. Par A. Sicé - Bale 722 
Larves d'onchoeerca volvulus dans le cristallin. Par M. Appelmans - Louvain 733 Pigmentary Degeneration of the Retina after Quinine Intoxication. By

H. M. Dekking - Groningen 743

Rétinite pigmentaire après maladies à virus. Par G. Maeder — Geneve . . 746 Amblyopie tabagique et Atrophie optique Hérédo-Familiale. Par L. Ham-

bresin et C. L. Schepens — Bruxelles 751

Retrobulbar Neuritis due to Malnutrition. By S. de Grósz - Budapest . . 764

214

Index

Untersuchungen an früher internierten Holländern. Von V. Stöckli-Bay,

A. Sícé, A. Perret-Gentíl und R. Bruckner - Basel 775

Missbîldungen und erbliche Augenleiden

La maladie de Sturge-Weber-Krabbe. Par J. D. Blum et S. Mutrux - Geneve 781

* Dysostose mandibulo-faciale unilatérale avec deformations multiples dnsquelette (processus paramastoïde, synostose des vertèbres, sacrali-sation etc.) et torticolis clonique. Par A.

Franceschetti, J.E. W.Brocheret D. Klein - Geneve 796

Dysplasie marginale postérieure de la cornée dans le cadre des malformations irido-cornéenne.

Par E. B. Streíff - Lausanne 815

Gross Remnants of the Pupillary Membrane, Anterior Polar Cataract andMícrocornea in a

Mother and her Children. By P. J. Waardenburg Arnhem 828

Beitrag zur Kenntnis der Menibrana epipapillaris. Von A. E. Schmid - Zurich 843

!!! A propos d'une atrophia gyrata choroideae et retinae de Fuchs apparais-sant dans une famille de rétinites pigmentaires. Par J. Bourquin $\uparrow$ etJ. B. Bourquin - Geneve 848

Beitrag zur Kasuistik der dominanten Vererbung der Retinitis pigmentosa.Von R. Heuscher-Isler - Basel, W. Gysin · Liestal und H. Hegner -St. Gallen 858

Operative und medikamentöse Therapie

The intra-capsular Cataract Operation - Experiences and Suggestions. By

M. Vannas - Helsinki 866

Les autoplasties par glissement dans les restaurations palpebrales. Par

R. de Saint-Martin - Toulouse $\quad 879$

Les kératites tardives par ypérite: Traitement par la greffe de conjonctive.

Par G.-P.Sourdille - Nantes 893

$7 / 8$ ' Le pronostic des greffes cornéennes. Par A. Franceschetti et V. Bischler -

Geneve $\quad 909$

Leber die operative Versorgung von frischen Wunden des Augapfels und

der Lider. Von H. K. Müller — Bonn 924

De Гemploi de Гacide pantothénique en ophtalmologie. Par L. Rosen - La

Chaux-de-Fonds $\quad 940$

Soziale Fürsorge

* $\quad$ Vereinfachung des Lesens Sehschwacher und Blinder. Von E. Kraupa Liberec 945

* Die Verminderung des Sehvermögens der Einäugigen im Sozialversiche-

rungsrecht. Von 7. Herzfeld - Basel 949

Vergleiфhende Ophthalmologie

Ueber den ophthalmoskopischen Nachweis von Fovea und Area bei Tieren. 
Die mit einem * aufgeführten Arbeiten sind nach dem 24. 8. 1947, zum Teil erst im Laufe des Jahres 1948, eingereicht warden. V.R. Von R. Bruckner - Basel 969 